\title{
Diminished Non-Classical Monocytes in the Blood Associate with Disease Severity in Alcoholic Hepatitis
}

\author{
Elisabeth Busk Rasmussen' \\ Lotte Lindgreen Eriksen ${ }^{2}$ \\ Stinne Ravn Greisen' \\ Anne Louise Hansen' \\ Mikkel Carstensen' \\ Thomas Damgaard Sandahl ${ }^{2}$ \\ Sidsel Støy ${ }^{2}$ \\ Tue Wenzel Kragstrup $\mathbb{D}^{1,3}$ \\ 'Department of Biomedicine, Aarhus \\ University, Aarhus, Denmark; \\ ${ }^{2}$ Department of Hepatology and \\ Gastroenterology, Aarhus University \\ Hospital, Aarhus, Denmark; ${ }^{3}$ Diagnostic \\ Center, Silkeborg Regional Hospital, \\ Silkeborg, Denmark
}

\begin{abstract}
Objective: Alcoholic hepatitis $(\mathrm{AH})$ holds a high mortality, and vast macrophage infiltration of the liver is involved in the progressive liver injury. No efficient medical treatment exists, and macrophages may be a future treatment target. Here, we examine associations between non-classical monocyte subsets and cell surface markers of migration with disease activity in patients with severe AH.

Methods: We analyzed samples from two cohorts of patients with AH. Cohort 1 included 15 $\mathrm{AH}$ patients, followed for 30 days, and 8 healthy controls (HCs). Cohort 2 included $23 \mathrm{AH}$ patients, followed for 90 days, and 9 HCs. Peripheral blood mononuclear cells (PBMCs) from both cohorts were analyzed by flow cytometry. Liver biopsies from cohort 2 were analyzed by RNA sequencing.

Results: Circulating non-classical monocytes in all but absent in patients with AH compared to $\mathrm{HC}$ in both cohorts (both $\mathrm{p}<0.0001$ ). The frequency of non-classical monocytes was significantly associated with Maddrey's discriminant function (mDF) $(r=-0.79, \mathrm{p}=0.0008$, cohort 1), Child-Pugh score (CP) $(r=-0.56, \mathrm{p}=0.03$, cohort 1), Model for End-Stage Liver Disease (MELD) $(r=-0.54, \mathrm{p}=0.02$, cohort 2) and C-reactive protein (CRP) $(r=-0.51$, $\mathrm{p}=0.027$, cohort 2). The surface expression of $\mathrm{CD} 11 \mathrm{~b}$ was increased on non-classical monocytes in patients with $\mathrm{AH}$ compared to $\mathrm{HC}(\mathrm{p}<0.0001)$ (cohort 1). The mRNA expression of $\mathrm{CD} 11 \mathrm{~b}$ was increased in liver biopsies in patients with $\mathrm{AH}$ compared to $\mathrm{HC}$ (cohort 2) $(\mathrm{p}<0.0001)$.
\end{abstract}

Conclusion: In this study, we describe an almost complete depletion of circulating nonclassical monocytes in the blood in two independent cohorts of patients with AH, which may be associated with a possible harmful recruitment of these cells to the liver. These results contribute to a better understanding of the disease, which hopefully can lead to therapies that target the acute inflammatory response leading to severe $\mathrm{AH}$.

Keywords: monocytes, non-classical, alcoholic hepatitis, CD11b, CCR2, CX3CR1

\section{Introduction}

Alcoholic hepatitis (AH) is an acute and severe hepatic inflammation associated with high mortality, and no efficient medical treatment exists. ${ }^{1}$ In order to develop a more efficient treatment of $\mathrm{AH}$, a better understanding of the pathogenesis and inflammatory processes is needed.

Circulating monocytes can be divided into three functionally distinct monocyte subsets based on the surface expression of CD14 and CD16. The non-classical monocyte subset $\left(\mathrm{CD} 14^{\text {low }} \mathrm{CD} 16^{\mathrm{pos}}\right)$ is the numerically smallest subset. However,
Correspondence: Tue Wenzel Kragstrup Department of Biomedicine, Aarhus University, Wilhelm Meyers Allé 4,

Aarhus C, DK-8000, Denmark

Tel +4587I67265

Email kragstrup@biomed.au.dk 
non-classical monocytes can greatly increase in number during inflammatory conditions such as sepsis, arthritis or multiple sclerosis. ${ }^{2}$ They are believed to act as patrolling cells surveilling the vessel walls and removing debris from the vasculature. ${ }^{3}$ In addition, they produce high levels of pro-inflammatory cytokines such as tumor necrosis factor (TNF)- $\alpha$ and interleukin (IL)- $1 .{ }^{4}$ Intermediate monocytes $\left(\mathrm{CD} 14^{\text {high }} \mathrm{CD} 16^{\text {pos }}\right)$ are poorly described, but also seem to produce pro-inflammatory cytokines and be highly phagocytic. ${ }^{5,6}$ Classical monocytes $\left(\mathrm{CD} 14^{\text {high }} \mathrm{CD} 16^{\text {neg }}\right)$ are the most abundant subset in peripheral blood and may be considered as pro-inflammatory cells expressing high levels of C-C motif chemokine receptor (CCR)-2 and producing pro-inflammatory cytokines upon stimulation.

During liver injury, hepatic macrophages become activated, which leads to inflammatory cytokine and chemokine release. This facilitates monocytes from the blood to migrate into the liver and supplement the Kupffer cells with monocyte-derived macrophages, increasing the total number of hepatic macrophages. ${ }^{7,8}$ In this process, the monocytes undergo phenotypic changes leading to a different expression of adhesion molecules on their cell surface. ${ }^{9}$ These adhesion molecules facilitate recruitment from the circulation to the liver tissue during AH. The integrin CD11b (Mac-1, CR3) is part of an integrin receptor, which activates downstream effects by allowing monocytes to adhere to endothelial cells and migrate through the vascular wall towards an inflamed area. ${ }^{9,10}$ This chemotaxis is also mediated by receptors such as $\mathrm{C}-\mathrm{C}$ motif chemokine receptor (CCR)-2 and C-X3-C motif chemokine receptor 1 (CX3CR1) found on the surface of several immune cells including monocytes. ${ }^{11}$ They regulate cell trafficking by binding to chemokines such as monocyte chemoattractant protein (MCP)-1, MCP-3, MCP-4, and Fractalkine (CX3RL1). ${ }^{5}$ CCR2 is highly expressed on classical monocytes whereas CX3CR1 is predominantly expressed by $\mathrm{CD} 16^{\text {pos }}$ monocytes, both intermediate and non-classical. ${ }^{11}$

Previously, an expansion of non-classical and intermediate monocytes has been shown in patients with chronic liver failure, both in circulation and even more in the liver. ${ }^{12}$ In contrast, a reduction in non-classical monocytes has been shown in patients with acute liver failure and in patients with $\mathrm{AH}^{6,13}$ A study including patients with alcoholic liver disease showed that the expression of MCP-1 was significantly increased both in serum and peripheral blood mononuclear cells (PBMCs) from $\mathrm{AH}$ patients compared with healthy controls (HCs). Also, the study showed an increased expression of CCR2 in liver biopsies from patients with alcoholic cirrhosis. ${ }^{14}$

Here, we confirm previous findings of diminished circulating non-classical monocytes in patients with severe $\mathrm{AH}$ in two independent cohorts. The reduction in nonclassical monocytes was associated with more severe disease. We also demonstrate that $\mathrm{CD} 11 \mathrm{~b}$ surface expression is increased on all monocytes in $\mathrm{AH}$ and that $\mathrm{CD} 11 \mathrm{~b}$ mRNA expression is increased in the liver in AH. These new findings may lead to a better understanding of the pathogenesis of $\mathrm{AH}$.

\section{Materials and Methods Study Subjects}

Two cohorts were included in this prospective cohort study. Both cohort 1 and cohort 2 have previously been thoroughly described. ${ }^{15,16}$

Briefly, cohort 1 consisted of $15 \mathrm{AH}$ patients and 8 age-matched HCs. Patients were recruited from four Danish hospitals in the time period 2008-2011 and followed for 30 days. Blood samples were obtained on the day of their diagnosis. Five patients died between time of diagnosis and day 30, and 13 patients were lost to follow-up or denied ongoing participation (Table 1).

Briefly, cohort 2 consisted of $23 \mathrm{AH}$ patients and 9 age-matched HCs. Patients were recruited from a single center (the Department of Hepatology and Gastroenterology, Aarhus University Hospital) in the time period 2013-2018. These patients were followed for 90 days and blood samples were obtained on the day of their diagnosis, and at day 7, and 90 after diagnosis. Further, a diagnostical liver biopsy was performed. Five patients died between their diagnosis and day 90 (Table 1). All patients were stratified using the Glasgow Alcoholic Hepatitis Score (GAHS) and were treated with nutritional support. Patients with GAHS $\geq 9$ were additionally treated with $400 \mathrm{mg} \times 3$ /day pentoxifylline or $40 \mathrm{mg} /$ day prednisolone in accordance with the Danish national guidelines at time of recruitment. On day 0 , this was 10 patients in cohort 1 and 12 patients in cohort 2 . In cohort 2, this was 8 patients on day 7 and 0 patients on day 90 .

\section{Inclusion and Exclusion Criteria}

In both cohorts, inclusion criteria followed international guidelines for inclusion of patients with $\mathrm{AH}$ in clinical 
Table I Patient Characteristics from Cohort I and Cohort 2

\begin{tabular}{|c|c|c|c|c|}
\hline & \multirow{2}{*}{$\frac{\text { Cohort I }}{\text { Day } 0}$} & \multicolumn{3}{|c|}{ Cohort 2} \\
\hline & & Day 0 & Day 7 & Day 90 \\
\hline Total patients $(\mathrm{n})$ & 15 & 23 & 18 & 10 \\
\hline Male (n) & 12 & 17 & 13 & 6 \\
\hline Female (n) & 3 & 6 & 5 & 4 \\
\hline Age (years) & $52 \pm 7$ & $55 \pm 11$ & $56 \pm 10$ & $57 \pm 11$ \\
\hline Weight (kg) & $73 \pm 17$ & - & - & - \\
\hline BMI $\left(\mathrm{kg} / \mathrm{m}^{2}\right)$ & $24 \pm 4$ & - & - & - \\
\hline ALT (I0-45 U/:) & $69 \pm 94$ & $55 \pm 39$ & $79 \pm 40$ & $36 \pm 19$ \\
\hline Bilirubin $(5-25 \mu \mathrm{mol} / \mathrm{L})$ & $303 \pm 176$ & $255 \pm 154$ & $226 \pm 166$ & $28 \pm 20$ \\
\hline $\operatorname{ALP}(10-75 \mathrm{U} / \mathrm{l})$ & $225 \pm 97$ & $230 \pm 119$ & $255 \pm 172$ & $|5| \pm 70$ \\
\hline Sodium (I37-145 mmol/L) & $|3| \pm 5$ & - & - & - \\
\hline Creatinine $(45-90 \mu \mathrm{mol} / \mathrm{L})$ & $75 \pm 28$ & $114 \pm 39$ & $102 \pm 96$ & $77 \pm 38$ \\
\hline Albumin $(36-45 \mathrm{~g} / \mathrm{l})$ & $28 \pm 6$ & $23 \pm 5$ & $25 \pm 6$ & $33 \pm 6$ \\
\hline Hemoglobin $(7.3-9.5 \mathrm{mmol} / \mathrm{L})$ & $6.8 \pm 0.9$ & $6.5 \pm 1.3$ & $6.6 \pm 1.1$ & $7.5 \pm 1.1$ \\
\hline Thrombocytes $\left(165-400 \times 10^{9} / \mathrm{L}\right)$ & $|4| \pm 68$ & $158 \pm 118$ & $211 \pm 203$ & $147 \pm 74$ \\
\hline INR $(<1.2)$ & $2 \pm 0.6$ & $1.8 \pm 0.4$ & $1.7 \pm 0.7$ & $1.3 \pm 0.2$ \\
\hline C-reactive protein $(\mathrm{mg} / \mathrm{L})$ & $39 \pm 14$ & $3 I \pm 23$ & $24 \pm 20$ & $4 \pm 3$ \\
\hline Leukocytes $\left(3.5-10.0 \times 10^{9} / \mathrm{L}\right)$ & $14.2 \pm 7.5$ & $11.4 \pm 7$ & $13 \pm 7.9$ & $4.2 \pm 3.7$ \\
\hline Monocytes $\left(0.20-0.70 \times 10^{9} / \mathrm{L}\right)$ & $1.7 \pm 0.6$ & $1 \pm 0.6$ & $0.9 \pm 0.6$ & $0.9 \pm 0.3$ \\
\hline MELD & $22 \pm 6.8$ & $22.2 \pm 9.2$ & $20.6 \pm 11.1$ & $9.5 \pm 6.9$ \\
\hline Child-Pugh Score & $\mid I . I \pm I .1$ & $10.6 \pm 1.3$ & $9.9 \pm 1.8$ & $6.4 \pm 0.7$ \\
\hline GAHS & $9.1 \pm 1.7$ & $8.4 \pm 1.6$ & $8.3 \pm 1.7$ & $6.5 \pm 0.9$ \\
\hline MDF & $76 \pm 34$ & - & - & - \\
\hline Fatal outcome (n) & 5 & - & - & 5 \\
\hline
\end{tabular}

Note: Data expressed as mean $\pm \mathrm{SD}$.

Abbreviations: BMI, body mass index; ALT, alanine aminotransferase; ALP, alkaline phosphatase; INR, international normalized ratio; MELD, Model for End-Stage Liver Disease; GAHS, Glasgow Alcoholic Hepatitis Score; MDF, Maddrey's discriminant function.

trials. ${ }^{17}$ The diagnosis was based on acute jaundice developed within the previous 2 weeks with serum bilirubin $>80$ $\mu \mathrm{mol} / \mathrm{L}$, clinical and biochemical variables as well as a history of excessive alcohol consumption with less than 3 weeks of abstinence leading up to the hospital admission. Patients were excluded if they had biliary stones, other liver diseases, gastrointestinal bleeding, signs of uncontrolled infection, any malignancy or if they had received immune-modulating therapy within 8 weeks before admission.

\section{Blood Sampling}

Venous blood samples were obtained from all patients and controls in EDTA vacuum tubes. Ficoll-Hypaque centrifugation was used to isolate PBMCs. PBMCs from both patients with $\mathrm{AH}$ and $\mathrm{HCs}$ were cryopreserved at $-140^{\circ} \mathrm{C}$.

\section{Flow Cytometric Analysis}

Flow cytometry was used to analyze the PBMCs and to divide them into three monocyte subsets based on their expression of CD14 and CD16; classical 
$\left(\mathrm{CD} 14^{\text {high }} \mathrm{CD} 16^{\text {neg }}\right)$, non-classical $\left(\mathrm{CD} 14^{\text {low }} \mathrm{CD} 16^{\text {pos }}\right)$ and intermediate $\left(\mathrm{CD} 14^{\mathrm{high}} \mathrm{CD} 16^{\mathrm{pos}}\right)$. In cohort 1 , the PBMCs were blocked with a buffer of PBS $+1 \%$ BSA $+10 \mu \mathrm{g} / \mathrm{mL}$ mouse IgG (Jackson ImmunoResearch, PA, USA) and incubated on ice for 10 minutes. PBMCs were first stained with LiveDead near-IR (Near-IR, Invitrogen) and washed. Then, the cells were stained with primary antibodies. The following primary antibodies were used: anti-CD14 V500 (BD Horizon, clone M $\varphi \mathrm{P} 9$ ), anti-CD16 PE/Cy7 (BioLegend, clone B73.1), anti-CD11b APC (BD Pharmingen, ICRF44), anti-CCR2 PerCP/Cy5.5 (BioLegend, clone $\mathrm{K} 036 \mathrm{C} 2$ ), and anti-CD56 APCeFlour780 (eBioscience, clone CMSSB). Anti-CD56 and LiveDead staining were combined in a dump channel to exclude dead cells and natural killer (NK) cells. In cohort 2, the PBMCs were blocked with $0.1 \mathrm{mg}$ human immunoglobulin (Privigen, CSL Behring, Denmark) pr. $\mathrm{mL}$ cell suspension and incubated for $15 \mathrm{~min}$ at $4{ }^{\circ} \mathrm{C}$ surface staining was performed using the following antibodies: anti-CD14-FITC (Miltenyi, cat. number 130-110-518), anti-CD16-APC-Vio770 (Miltenyi, cat. number 130-096655), anti-Gal9-BV421 (BioLegend cat. number 348,920), anti-4-1BBL-APC (Miltenyi, cat. number 130-103-656), anti-Gal3-PE (Miltenyi, cat. number 130-101-315) and viability-viogreen (Miltenyi, cat. number 130-110-206). Gating strategy is shown in Figure S1.

Data were analyzed in FlowJo (Tree Star Inc., Ashland, OR, USA). For cohort 1, the analysis was done on BD Fortezza and for cohort 2, the analysis was done on Macs Quant Analyzer 10. The migration markers CD11b and CCR2, were only measured in samples from patients in cohort 1.

\section{Liver RNA Sequencing}

RNA sequencing was used to measure liver mRNA expression on liver biopsies as previously described ${ }^{16}$ and compared with biopsies from the healthy rim of liver resectates during surgery for metastatic colon cancer.

\section{Statistics}

GraphPad Prism 8.4.0 was used to perform statistical analyses. Non-parametric tests were used depending on whether data were normally distributed. All results are presented as median and interquartile range. Spearman's rho was used for correlation analyses. The results were considered statistically significant with a two-tailed p-value below 0.05 .

\section{Results}

The Percentage of Non-Classical Monocytes in Peripheral Blood is Decreased in $\mathrm{AH}$

In cohort 1 , the median percentage of non-classical monocytes was $0.55 \%$ (interquartile range $0.38-1.4 \%$ ) for $\mathrm{AH}$ patients and $3.6 \%$ (interquartile range $2.2-11.0 \%$ ) for $\mathrm{HC}$ $(\mathrm{p}=0.0001)$ (Figures 1A and B). The percentage of classical and intermediate monocytes did not differ between patients and $\mathrm{HC}$ (Figures $1 \mathrm{~A}$ and $\mathrm{B}$ ). In cohort 2, the median percentage of non-classical monocytes was $0.64 \%$ (interquartile range $0.4-1.6 \%$ ) for $\mathrm{AH}$ patients and $3.2 \%$ (interquartile range $1.6-5.0 \%$ ) for $\mathrm{HC}$ at diagnosis $(\mathrm{p}=0.0001)$. At day 7 , the median percentage was $0.35 \%$ (interquartile range $0.12-0.94 \%$ ) for $\mathrm{AH}$ patients and 3.2\% (interquartile range $1.6-5.0 \%)$ for $\mathrm{HC}(\mathrm{p}<0.0001)$ (Figure $1 \mathrm{C})$. The percentage of classical and intermediate monocytes did not differ at any study day (Figure 1C).

\section{The Percentage of Non-Classical Monocytes Associates with Disease Severity in $\mathrm{AH}$}

In cohort 1, the percentage of non-classical monocytes was inversely associated with Maddrey's discriminant function $(\mathrm{mDF})(r=-0.79, \mathrm{p}=0.0008)$ and Child-Pugh score (CP) $(r=-0.56, \mathrm{p}=0.03) \quad$ (Table 2). No associations were observed between the percentage of classical or intermediate monocytes and any disease activity score. In cohort 2 , the percentage of non-classical monocytes was inversely associated with Model for End-Stage Liver Disease (MELD) $(r=-0.54, \mathrm{p}=0.02)$ and C-reactive protein (CRP) $(r=-0.51, \mathrm{p}=0.027)$ (Table 2$)$. The frequency of intermediate monocytes in cohort 2 correlated inversely with GAHS $(r=-0.46, \mathrm{p}=0.05)$ and $\mathrm{CP}(r=-0.49, \mathrm{p}=0.04)$. The percentage of classical monocytes from cohort 2 did not correlate with any disease score (Table 2).

\section{The Surface Expression of CDIIb is Increased on Non-Classical Monocytes in $\mathrm{AH}$}

The surface expression of the integrin receptor CD11b was increased on non-classical monocytes in $\mathrm{AH}$ patients compared to HC $(p<0.0001)$ (Figure 2). The surface expression of CCR2 was not differently expressed on non-classical monocytes. On intermediate monocytes, the surface expression of both CD11b $(\mathrm{p}<0.0001)$ and CCR2 
A

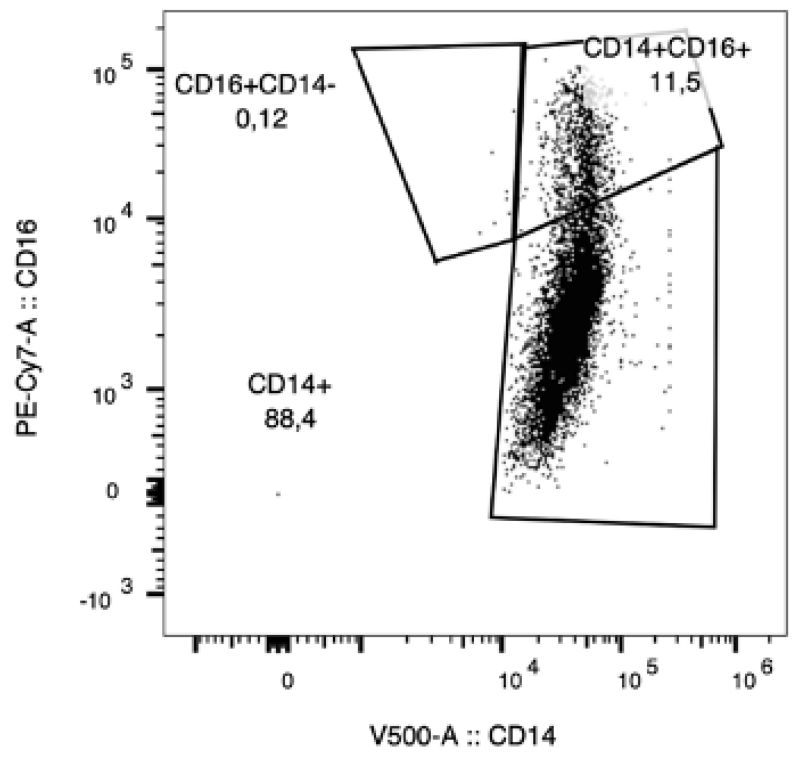

B Non-classical monocytes

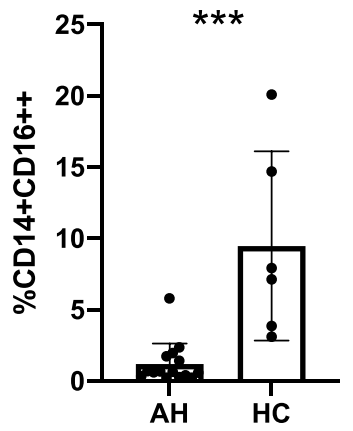

C Non-classical monocytes

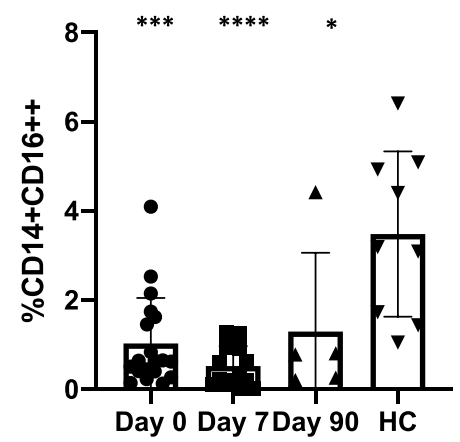

Intermediate monocytes

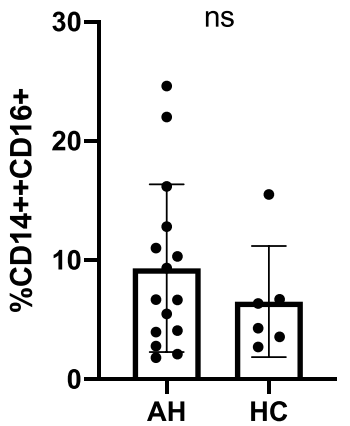

Intermediate monocytes

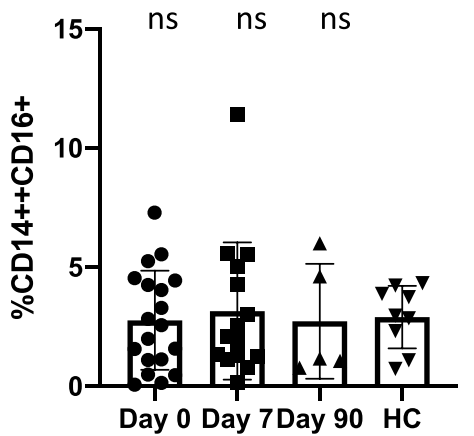

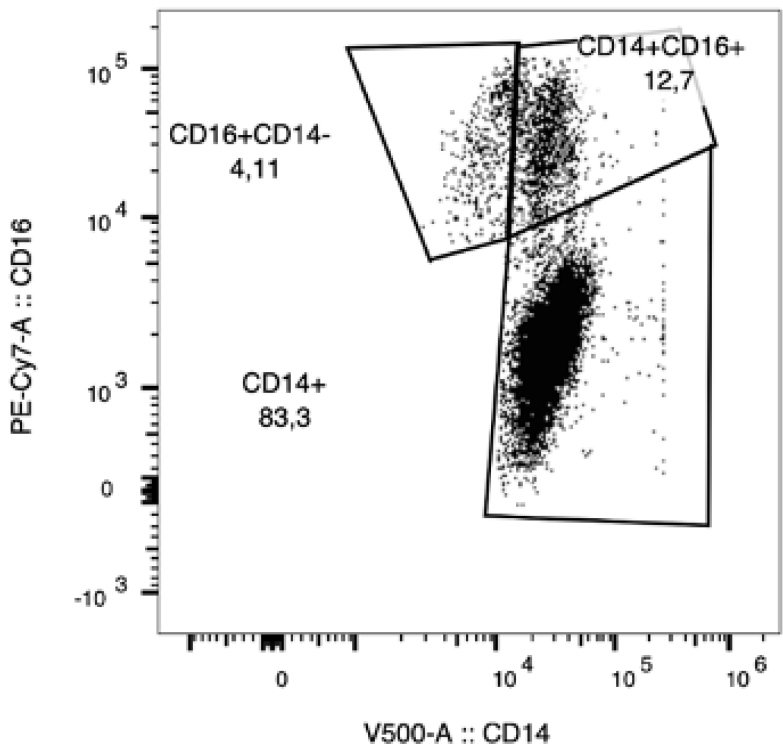

Classical monocytes

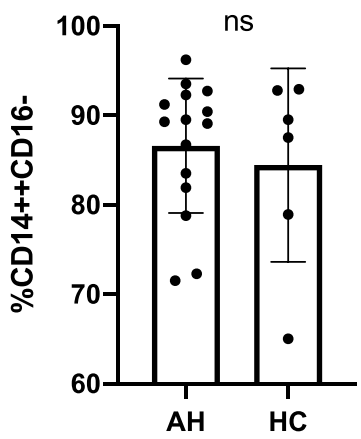

Classical monocytes

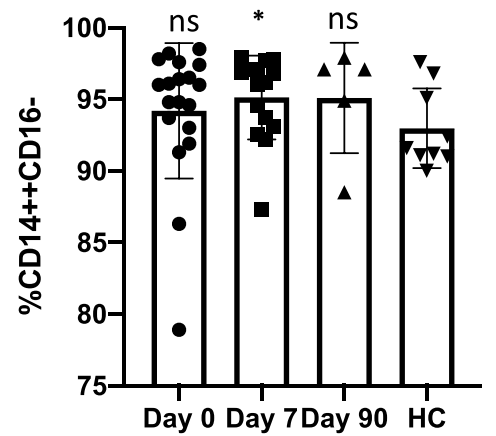

Figure I The peripheral blood cells were analyzed using flow cytometry to measure the CDI4 and CDI6 expression on monocyte subsets from cohort I (A and B). Surface expression of CDI4 and CDI6 on monocytes from $\mathrm{AH}$ patients at day of diagnosis and 7 and 90 days after diagnosis and from $\mathrm{HC}$ from cohort 2 (C). The monocytes were divided into three subsets based on their expression of CDI4 and CDI6 (classical CDI4 ${ }^{++} \mathrm{CD} / 6^{-}$, intermediate $\mathrm{CDI} 4^{++} \mathrm{CDI} 6^{+}$and non-classical $\mathrm{CDI} 4^{+} \mathrm{CDI} 6^{++}$). Data are presented as median with range, $\mathrm{n}=\mathrm{I} 5$ (cohort $\mathrm{I}$ ) and $\mathrm{n}=23$ (cohort 2 ), asterisk $(*)$ marks a significant correlation and $\mathrm{p}$ value $<0.05,(* * *) p<0.00 \mathrm{I}$, $(* * * *) p<0.0001$

Abbreviations: $\mathrm{AH}$, alcoholic hepatitis patients; $\mathrm{HC}$, healthy controls; ns, non-significant.

$(\mathrm{p}=0.0007)$ were upregulated. On classical monocytes, neither CD11b nor CCR2 were upregulated in AH patients compared with $\mathrm{HC}$ (Figure 2). No associations were observed between the surface expression of CD11b or CCR2 on non-classical, intermediate and classical monocytes and any disease activity score (Table S1). 
Table 2 Correlations Between the Frequency of Non-Classical, Intermediate and Classical Monocyte Subsets and Disease Scores

\begin{tabular}{|l|l|l|l|l|l|l|}
\hline & \multicolumn{3}{|c|}{ Cohort I } & \multicolumn{3}{c|}{ Cohort 2 } \\
\cline { 2 - 7 } & Non-Classical & Intermediate & Classical & Non-Classical & Intermediate & Classical \\
\hline mDF & $-0.79(0.0008)^{*}$ & $0.13(0.65)$ & $-0.01(0.96)$ & - & - & - \\
\hline MELD & $-0.46(0.08)$ & $0.50(0.06)$ & $-0.36(0.19)$ & $-0.54(0.02)^{*}$ & $-0.25(0.32)$ & $0.26(0.30)$ \\
\hline GAHS & $-0.46(0.10)$ & $0.26(0.36)$ & $-0.18(0.53)$ & $-0.42(0.08)$ & $-0.46(0.05)^{*}$ & $0.24(0.33)$ \\
\hline CP & $-0.56(0.03)^{*}$ & $0.06(0.84)$ & $-0.06(0.84)$ & $-0.22(0.39)$ & $-0.49(0.04)^{*}$ & $0.30(0.22)$ \\
\hline CRP & $-0.09(0.76)$ & $-0.32(0.26)$ & $0.20(0.50)$ & $-0.51(0.03)^{*}$ & $-0.16(0.51)$ & $-0.01(0.96)$ \\
\hline
\end{tabular}

Notes: Data assessed by Spearman correlation and presented as correlation coefficient, $r$ and $f(p)$. Asterix $(*)$ marks a significant correlation.

Abbreviations: mDF, Maddrey's discriminant function; MELD, Model for End-Stage Liver Disease; GAHS, Glasgow Alcoholic Hepatitis Score; CP, Child-Pugh Score.

The Expression of CDIIb and CX3CR I mRNA is Increased in the Liver in $\mathrm{AH}$

The expression of CD11b was significantly increased in AH patients $(p<0.0001)$ while the increase in CX3CR1 was not significant $(\mathrm{p}<0.054)$ (Figure 3). The expression of CD14, CD16a, CD16b or CCR2 did not differ between $\mathrm{AH}$ patients and controls. The expression of CD16b correlated positively with $\mathrm{CP}(\mathrm{r}=0.62, \mathrm{p}=0.03)$ (Table $\mathrm{S} 2)$. No
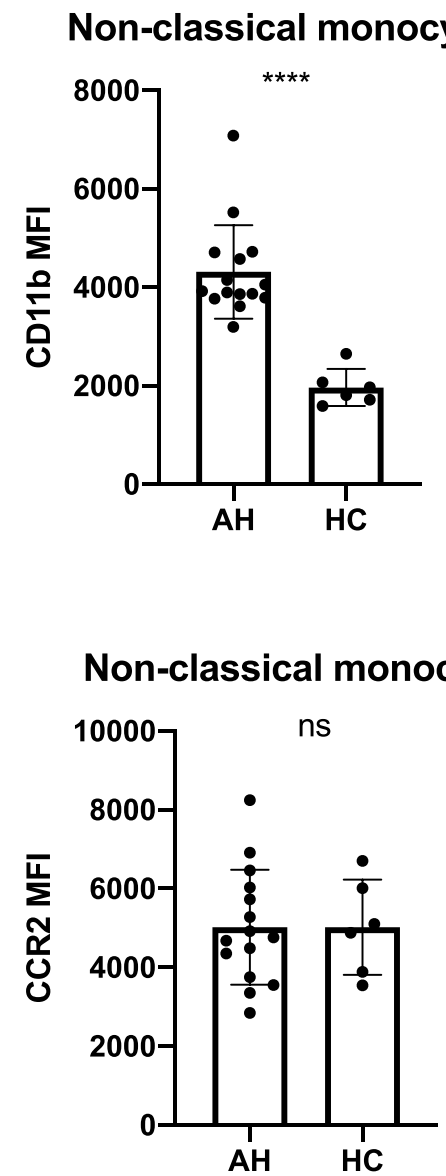

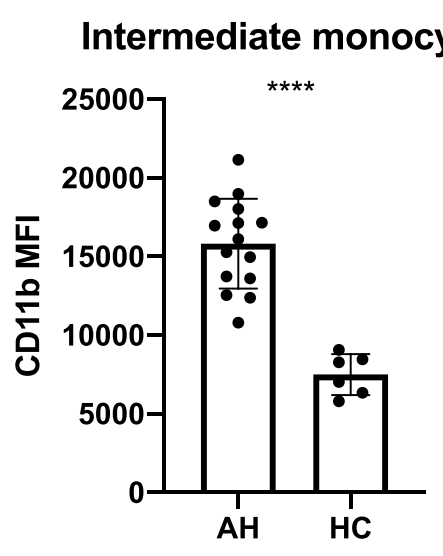

Intermediate monocytes

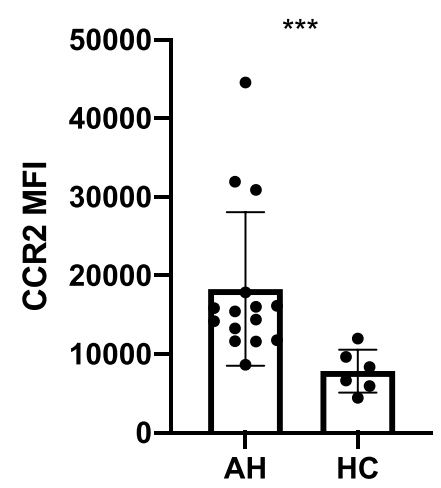

\section{Classical monocytes}

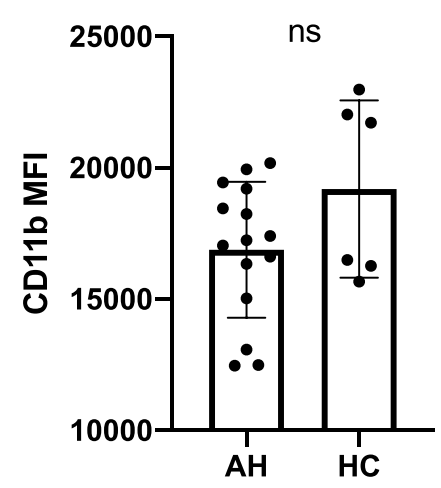

Classical monocytes

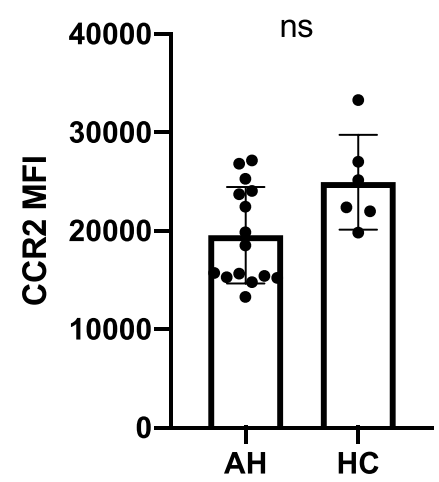

Figure 2 Cohort I: Surface expression of CDI Ib and CCR2 on monocyte subsets in AH patients and HC, measured by flow cytometry. Monocyte subsets are defined as (classical CDI4 ${ }^{++} \mathrm{CDI} 6^{-}$, intermediate $\mathrm{CDI} 4^{++} \mathrm{CDI} 6^{+}$and non-classical CDI4 $\left.4^{+} \mathrm{CDI} 6^{++}\right)$. Data are presented as median with range, $\mathrm{n}=15(\mathrm{cohort} \mathrm{I})$. Triple asterisk $(* * *)$ marks a significant correlation and $p$ value $p<0.00 \mathrm{I},(* * * *) p<0.000 \mathrm{I}$.

Abbreviations: $\mathrm{AH}$, alcoholic hepatitis patients; $\mathrm{HC}$, healthy controls; ns, non-significant. 


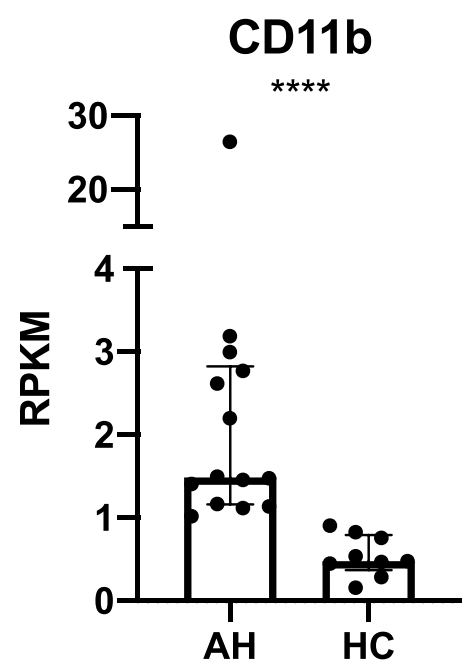

CX3CR1

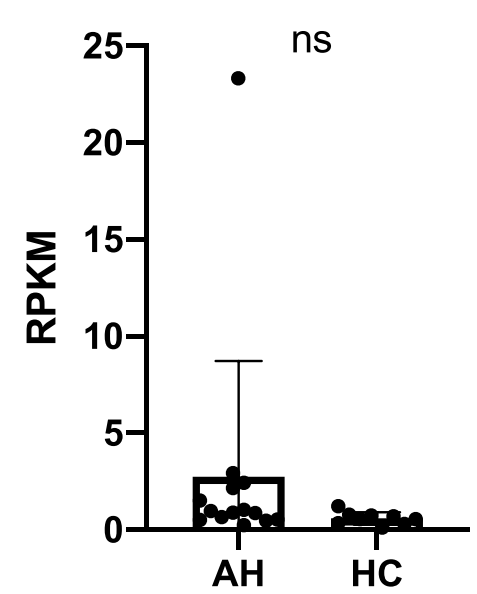

CD14

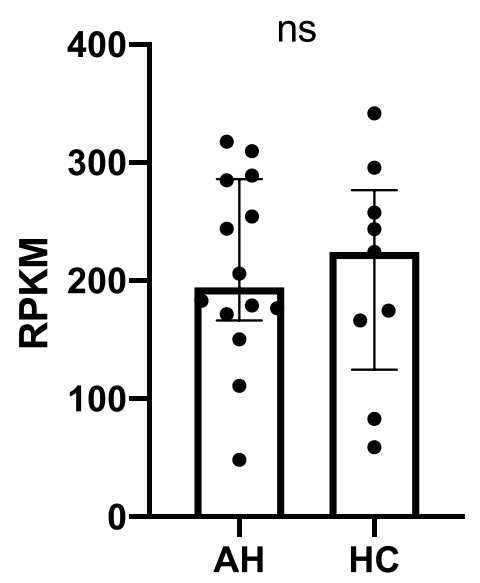

CD16a

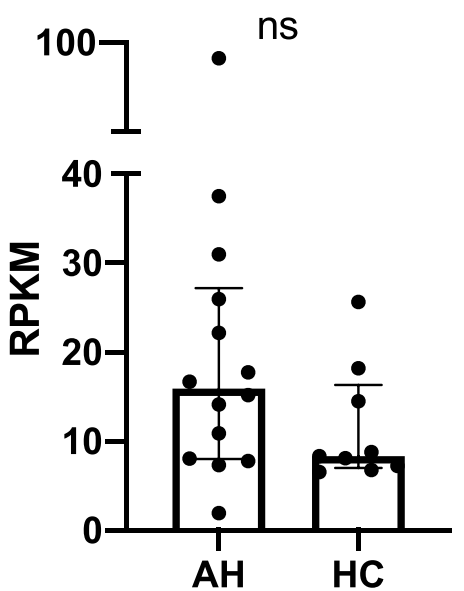

CCR2

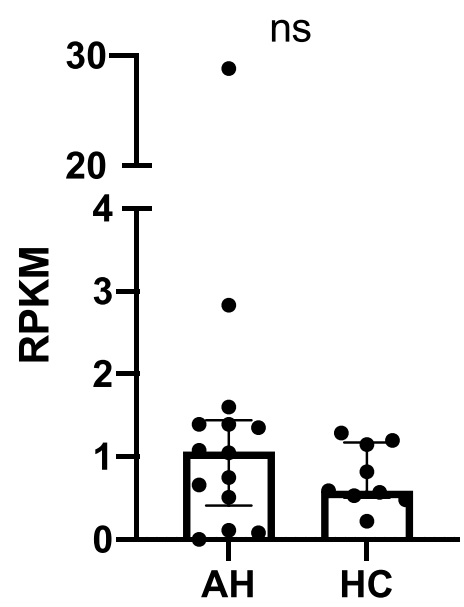

CD16b

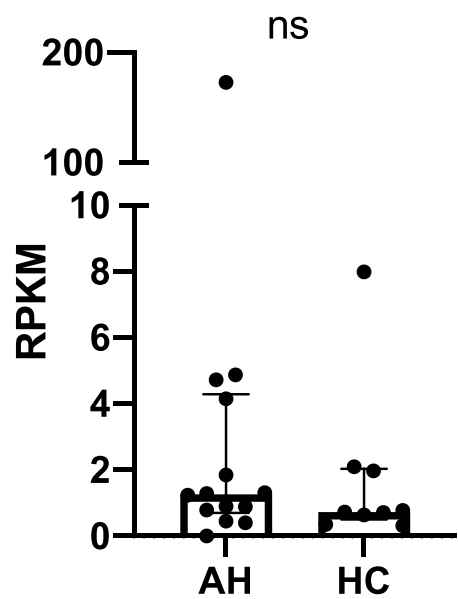

Figure 3 Cohort 2: The expression of CDI Ib, CX3CRI, CCR2, CD I4, CDI6a and CDI6b analyzed by RNA sequencing of liver biopsies from AH and HC on day 0. Data are presented as median with interquartile range. $\mathrm{n}=23$. Quadruple asterisk $(* * * *)$ marks a significant correlation and $\mathrm{p}$ value $<0.000 \mathrm{I}$.

Abbreviations: AH, alcoholic hepatitis patients; HC, healthy controls; ns, non-significant; RPKM, reads per kilobase million.

other associations were observed between disease activity scores and the mRNAs (Table S2).

\section{Discussion}

Monocytes and macrophages play a pivotal role in the hepatic inflammatory response during AH. However, the pathogenesis is still to be fully understood, and a targeted medical treatment is lacking. In this study, we report a significant and constant reduction in non-classical monocytes in $\mathrm{AH}$ patients, and the percentage of non-classical monocytes correlated inversely with disease activity scores.

There are several limitations to this study. First, we did not include patients with other liver diseases for comparison. Therefore, it is not known if our observations are specific to alcoholic hepatitis. Second, FMOs and Slan staining could have improved the precision of the monocyte subset gating. However, gates were drawn similarly in samples from patients with AH and HCs. Therefore, differences between patients with $\mathrm{AH}$ and HCs were likely not caused by differences in gating strategy. Third, the cells were not measured together with counting beads. Therefore, changes in absolute cell numbers cannot be assessed. Finally, the primary antibodies used for the flow cytometric staining were different in the two cohorts. It is a strength that the findings despite the different use of antibodies are similar in the two cohorts. However, absolute cell numbers or cell subset proportions cannot be compared between the two cohorts. 
The overall concentration of monocytes in the liver increases during severe $\mathrm{AH} .{ }^{18-20}$ We have now confirmed previous findings of decreased percentages of non-classical monocytes in the blood. ${ }^{6,9}$ The frequency of non-classical monocytes correlated inversely with disease activity scores (mDF and CP for cohort 1; MELD for cohort 2). This further strengthens the association between diminished percentage of non-classical monocytes and acute $\mathrm{AH}$. In cohort 2, the patients who survive to day 90 improve their biochemistry and also increase their non-classical monocytes frequencies. However, non-classical monocyte numbers do not reach the level of the healthy controls. This probably reflects that some patients resume drinking excess alcohol and have underlying cirrhosis. This is accompanied by low-grade systemic inflammation and a susceptibility to infections. These are all factors that may hinder complete normalization of non-classical monocytes.

However, it is still not clear whether this association is causal and the potential direction of causality. The lack of non-classical monocytes in peripheral blood may be explained by the migration of these cells into the liver, or it may reflect a deficiency due to increased apoptosis or differentiation caused by inflammation. Further, the association could be explained by a simple increase in the classical monocyte subset changing the monocyte distribution. Here we studied co-expression of CD11b and CCR2 on the surface of monocyte subsets to examine whether changes in these surface membrane receptors is correlated to disease activity and the decrease in non-classical monocytes. The surface expression of $\mathrm{CD} 11 \mathrm{~b}$ was increased in both non-classical and intermediate monocyte subsets suggesting a priming for migration of especially these monocytes during inflammation in AH. CCR2-expression was increased on intermediate monocytes but not on non-classical monocytes from $\mathrm{AH}$ patients compared to $\mathrm{HC}$. There were no associations between the expression of CD11b or CCR2 on any monocyte subset and disease activity scores in AH. We further analyzed the mRNA expression of selected genes in liver biopsies to determine if the non-classical monocytes migrated into the liver. The mRNA expression of CD11b was increased in the liver of $\mathrm{AH}$ patients. This is in line with previous studies showing increased migration of $\mathrm{CD} 11 \mathrm{~b}$ expressing cells to the liver in $\mathrm{AH} .{ }^{19}$ In this study, CX3CR1 was also numerically increased in the liver of $\mathrm{AH}$ patients. Increased CX3CR1 has been shown on CD16+ monocytes. ${ }^{11,21}$ This substantiates the migration of non-classical monocytes to the liver in $\mathrm{AH}$. These results are in line with previous studies on end stage liver disease, showing accumulation of $\mathrm{CD} 16^{\text {pos }}$ monocytes in the liver. ${ }^{22}$ However, CD11b is also expressed by other myeloid cells and a contribution of local differentiation of classical to non-classical monocytes cannot be ruled out.

Alcoholic hepatitis is a serious disease caused by excessive, heavy drinking leading to inflammation of the liver causing high morbidity and mortality for the patients. To this day, no targeted medical treatment is available to cure this disease; thus, a better understanding of the pathogenesis is needed to bring us closer to developing a medical treatment. With this study, we have contributed with new knowledge about the pathogenesis of AH by showing a substantial decrease in non-classical monocytes correlating with disease activity scores. These data collectively suggest monocytes recruitment to the liver to promote disease progression in $\mathrm{AH}$ and may thus be a future treatment target.

\section{Abbreviations}

$\mathrm{AH}$, alcoholic hepatitis; $\mathrm{HC}$, healthy controls; $\mathrm{mDF}$, Maddrey's discriminant function; MELD, Model for EndStage Liver Disease; CCR, C-C motif chemokine receptor; CX3CR1, CX3C chemokine receptor 1; CRP, C-reactive protein; GAHS, Glasgow Alcoholic Hepatitis Score; CP, Child-Pugh Score; PP, performance points; TNF, tumor necrosis factor; IL, Interleukin; PBMCs, peripheral blood mononuclear cells; MCP, monocyte chemoattractant protein.

\section{Data Sharing Statement}

Please contact TWK for data requests.

\section{Ethics Approval and Consent to Participate}

Before being included in the study, the patients had completed a written consent according to the Declaration of Helsinki. The study was approved by the Central Denmark Region ethical committee. All patients were informed about the purpose of the study.

\section{Author Contributions}

All authors made substantial contributions to conception and design, acquisition of data, or analysis and interpretation of data; took part in drafting the article or revising it critically for important intellectual content; agreed to submit to the current journal; gave final approval of the version to be published; and agree to be accountable for all aspects of the work. 


\section{Funding}

TWK was supported by a grant from Independent Research Fund Denmark (9039-00015B).

\section{Disclosure}

TWK has engaged in educational activities receiving speaking fees from Pfizer, Bristol-Myers Squibb, Eli Lilly, Novartis, and UCB and has been consultant and advisor for Bristol-Myers Squibb and Gilead. TWK is co-owner and clinical developer in iBiotech ApS developing diagnostic and therapeutic solutions for people with autoimmune diseases and cancer. The other authors have no conflicts of interest.

\section{References}

1. Hosseini N, Shor J, Szabo G. Alcoholic hepatitis: a review. Alcohol Alcohol. 2019;54(4):408-416. doi:10.1093/alcalc/agz036

2. Mukherjee R, Kanti Barman P, Kumar Thatoi P, et al. Non-classical monocytes display inflammatory features: validation in sepsis and systemic lupus erythematous. Sci Rep. 2015;5(1). doi:10.1038/srep13886

3. Auffray C, Fogg D, Garfa M, et al. Monitoring of blood vessels and tissues by a population of monocytes with patrolling behavior. Science. 2007;317(5838):666-670. doi:10.1126/science.1142883

4. Ong S-M, Hadadi E, Dang T-M, et al. The pro-inflammatory phenotype of the human non-classical monocyte subset is attributed to senescence article. Cell Death Dis. 2018;9(3). doi:10.1038/s41419-018-0327-1

5. França CN, Izar MCO, Hortêncio MNS, et al. Monocyte subtypes and the CCR2 chemokine receptor in cardiovascular disease. Clin Sci. 2017;131(12):1215-1224. doi:10.1042/CS20170009

6. Waller K, James C, de Jong A, et al. ADAM17-mediated reduction in $\mathrm{CD} 14++\mathrm{CD} 16+$ monocytes ex vivo and reduction in intermediate monocytes with immune paresis in acute pancreatitis and acute alcoholic hepatitis. Front Immunol. 2019;10. doi:10.3389/fimmu.2019.01902

7. Tacke F. Targeting hepatic macrophages to treat liver diseases. J Hepatol. 2017;66(6):1300-1312. doi:10.1016/j.jhep.2017.02.026

8. Karlmark KR, Weiskirchen R, Zimmermann HW, et al. Hepatic recruitment of the inflammatory Gr1+monocyte subset upon liver injury promotes hepatic fibrosis. Hepatology. 2009;50(1):261-274. doi:10.1002/ hep. 22950

9. Cifarelli V, Libman IM, DeLuca A, et al. Increased expression of monocyte CD11b (Mac-1) in overweight recent-onset type 1 diabetic children. Rev Diabet Stud. 2007;4(2):112-120. doi:10.1900/RDS.2007.4.112

10. Subhi Y, Krogh Nielsen M, Molbech CR, et al. Association of CD11b + monocytes and anti-vascular endothelial growth factor injections in treatment of neovascular age-related macular degeneration and polypoidal choroidal vasculopathy. JAMA Ophthalmol. 2019;137(5):515. doi:10.1001/jamaophthalmol.2019.0010
11. Chen -Y-Y, Arndtz K, Webb G, et al. Intrahepatic macrophage populations in the pathophysiology of primary sclerosing cholangitis. JHEP Rep. 2019;1(5):369-376. doi:10.1016/j.jhepr.2019.10.003

12. Zimmermann HW, Seidler S, Nattermann J, et al. Functional contribution of elevated circulating and hepatic non-classical CD14 $+\mathrm{CD} 16+$ monocytes to inflammation and human liver fibrosis. PLoS One. 2010;5(6):e11049. doi:10.1371/journal.pone.0011049

13. Abeles RD, McPhail MJ, Sowter D, et al. CD14, CD16 and HLA-DR reliably identifies human monocytes and their subsets in the context of pathologically reduced HLA-DR expression by CD14hi/CD16neg monocytes: expansion of CD14hi/CD16pos and contraction of CD14lo/CD16pos monocytes in acute liver fail. Cytometry $A$. 2012;81A(10):823-834. doi:10.1002/cyto.a.22104

14. Ambade A, Lowe P, Kodys K, et al. Pharmacological inhibition of CCR2/5 signaling prevents and reverses alcohol-induced liver damage, steatosis, and inflammation in mice. Hepatology. 2019;69 (3):1105-1121. doi:10.1002/hep.30249

15. Støy S, Sandahl TD, Hansen AL, et al. Decreased monocyte shedding of the migration inhibitor soluble CD18 in alcoholic hepatitis article. Clin Transl Gastroenterol. 2018;9(6). doi:10.1038/s41424-018-00227

16. Støy S, Laursen TL, Glavind E, et al. Low interleukin-22 binding protein is associated with high mortality in alcoholic hepatitis and modulates interleukin-22 receptor expression. Clin Transl Gastroenterol. 2020;11(8):e00197. doi:10.14309/ ctg.0000000000000197

17. Crabb DW, Bataller R, Chalasani NP, et al. Standard definitions and common data elements for clinical trials in patients with alcoholic hepatitis: recommendation from the NIAAA alcoholic hepatitis consortia. Gastroenterology. 2016;150(4):785-790. doi:10.1053/j. gastro.2016.02.042

18. Sandahl TD, Grønbæk H, Møller HJ, et al. Hepatic macrophage activation and the LPS pathway in patients with alcoholic hepatitis: a prospective cohort study. Am J Gastroenterol. 2014;109 (11):1749-1756. doi:10.1038/ajg.2014.262

19. Wang M, You Q, Lor K, et al. Chronic alcohol ingestion modulates hepatic macrophage populations and functions in mice. $J$ Leukoc Biol. 2014;96(4):657-665. doi:10.1189/jlb.6A0114-004RR

20. Kim A, Bellar A, McMullen MR, Li X, Nagy LE. Functionally diverse inflammatory responses in peripheral and liver monocytes in alcohol-associated hepatitis. Hepatol Commun. 2020;4 (10):1459-1476. doi:10.1002/hep4.1563

21. Geissmann F, Jung S, Littman DR. Blood monocytes consist of two principal subsets with distinct migratory properties. Immunity. 2003;19(1):71-82. doi:10.1016/S1074-7613(03)00174-2

22. Liaskou E, Zimmermann HW, Li -K-K, et al. Monocyte subsets in human liver disease show distinct phenotypic and functional characteristics. Hepatology. 2013;57(1):385-398. doi:10.1002/ hep. 26016

\section{Publish your work in this journal}

Clinical and Experimental Gastroenterology is an international, peerreviewed, open access, online journal publishing original research, reports, editorials, reviews and commentaries on all aspects of gastroenterology in the clinic and laboratory. This journal is indexed on American Chemical Society's Chemical Abstracts Service (CAS).
The manuscript management system is completely online and includes a very quick and fair peer-review system, which is all easy to use. Visit http://www.dovepress.com/testimonials.php to read real quotes from published authors. 